\title{
Saúde mental infantil em contextos de desvantagem socioeconômica: fatores de risco e proteçáo ${ }^{1}$
}

\author{
Thelma Simóes Matsukuraa ${ }^{a}$ Amanda Dourado Souza Akahosi Fernandes ${ }^{b}$, \\ Maria Fernanda Barboza Cid ${ }^{\mathrm{c}}$
}

\begin{abstract}
${ }^{a}$ Departamento de Terapia Ocupacional, Programa de Pós-Graduação em Terapia Ocupacional, Programa de Pós-Graduação em Educação Especial, Universidade Federal de São Carlos - UFSCar, São Carlos, SP, Brasil bPrograma de Pós-Graduação em Terapia Ocupacional, Universidade Federal de São Carlos - UFSCar, São Carlos, SP, Brasil

'Departamento de Terapia Ocupacional, Universidade Federal de São Carlos - UFSCar, São Carlos, SP, Brasil
\end{abstract}

\begin{abstract}
Resumo: O objetivo do estudo foi identificar e comparar situações de risco ou proteção ao desenvolvimento socioemocional de crianças que vivem em contextos de desvantagem socioeconômica. Participaram desta pesquisa sete crianças com idade entre oito e dez anos e respectivos responsáveis. As crianças eram alunos do segundo ao quinto ano do ensino fundamental de uma escola pública do interior do estado de São Paulo. Para fins de comparação, os participantes foram divididos em dois grupos: um grupo, composto pelos responsáveis e suas crianças, foi avaliado pelo SDQ - Questionário de Capacidades e Dificuldades - com sintomas clínicos (GSC - Grupo com Sintomas Clínicos de Saúde Mental) e o outro, igualmente composto por crianças e seus responsáveis, mas cujas crianças não apresentavam sintomas clínicos segundo a mesma avaliação (GDT - Grupo com Desenvolvimento Típico). Para a coleta de dados foram utilizados dois roteiros de entrevistas, um apresentado à criança e outro ao responsável. Para a análise de dados utilizou-se a técnica do Discurso do Sujeito Coletivo (DSC). Os resultados apontaram tanto semelhanças quanto diferenças entre o GSC e o GDT. Em relação às semelhanças, as crianças possuem regras e responsabilidades e os pais buscam auxílio para o cuidado da criança através de equipamentos sociais, de saúde e educacionais. Quanto às diferenças, no GSC as crianças se referem à escola de maneira negativa e há menor auxílio dos pais nas atividades escolares. Aponta-se que estudos dessa natureza podem contribuir para o debate acerca de políticas públicas e práticas voltadas a essa população.
\end{abstract}

Palavras-chave: Saúde Mental, Fator de Risco, Família, Criança.

\section{Child mental health in socioeconomically disadvantaged contexts: risk and protective factors}

\begin{abstract}
The main objective of this study is to identify and compare different situations of risk or protection in the socio-emotional development of children living in socioeconomically disadvantaged contexts. Seven (7) children aged eight to ten and their respective parents participated in the present survey. The children were $2^{\text {nd }}$ to $5^{\text {th }}$ grade students at an elementary public school in the countryside of Sao Paulo state. The subjects involved in this survey were divided into two different groups: one composed of children evaluated by the Strengths and Difficulties Questionnaire (SDQ) - with clinical symptoms (Group with Clinical Symptoms of Mental Health - GSC); the other group was composed of children that did not present clinical symptoms (Group with Typical Development - GDT) and their parents. Two different interview scripts were used for data collection: one answered by the children and the other responded by the parents. Data analysis was based on the technique of Collective Subject Discourse (CSD). The results showed similarities and differences between the GSC and GDT groups. Regarding the similarities, all the children have rules and responsibilities, and all the parents seek assistance in the care of their children by means
\end{abstract}

Autor para correspondência: Thelma Simões Matsukura, Universidade Federal de São Carlos, Rod. Washington Luís, km 235, CEP 13565-905, São Carlos, SP, Brasil, e-mail: thelma@ufscar.br

Recebido em 20/12/2012; Revisão em 26/8/2013; Aceito em 23/9/2013. 
of social, health and educational services. Concerning the differences, children in the GSC group refer to school in a negative way and have less support from their parents in school activities. It is worth mentioning that studies of this nature can contribute to the debate on public policies and practices aimed at this population.

Keywords: Mental Health, Risk Factor, Family, Child.

\section{Introdução}

O desenvolvimento infantil é influenciado por características pessoais, físicas e mentais do indivíduo e pelo ambiente social em que ele vive, de forma que ameaças diretas ao desenvolvimento podem ocorrer como consequência de problemas genéticos e biológicos adquiridos e também pela presença de adversidades ambientais. Assim, os resultados negativos do desenvolvimento são produzidos numa interação complexa entre eles, tornando-se fatores de risco para o desenvolvimento saudável das crianças (HALPERN; FIGUEIRAS, 2004; BANDIN, 2008; KOLLER, 2004; LINHARES; BORDIM; CARVALHO, 2004).

De acordo com a literatura, riscos, nos indivíduos a ele expostos, são variáveis associadas a uma alta probabilidade de desenvolvimento de problemas de saúde mental, em diferentes níveis de severidade, além disso estáo associados a eventos negativos de vida, uma vez que aumentam a probabilidade e do indivíduo apresentar problemas, não sendo todavia fator determinante desse efeito (COIE et al., 1993; SAPIENZA; PEDROMÔNICO, 2005; HUTZ, 2005).

Quando muitas situaçóes de risco se associam, dificultam o processo de desenvolvimento, por isso a importância de se prever fatores de risco não está tanto relacionada a um prognóstico ruim, mas principalmente à demonstração da necessidade de ser capaz de intervir quando for preciso (SAPIENZA; PEDROMÔNICO, 2005).

Hutz (2005) aponta algumas situações como sendo de risco pessoal e social: nível socioeconômico baixo e condiçôes de miserabilidade; número grande de indivíduos na composição familiar; ausência dos pais e psicopatologias na família.

Assis, Avanci e Oliveira (2009), em estudo realizado com 479 crianças entre seis e 13 anos de escolas públicas de São Gonçalo, verificaram dentre os resultados que viver em condiçóes socioeconômicas muito precárias e ter pais com baixa escolaridade são fatores que se mostram relacionados com a precária competência social e a problemas de comportamento em crianças.

Outros estudos também apontam renda familiar baixa como fator de risco para problemas de saúde mental nas crianças. Ferriolli, Marturano e Puntel (2007) realizaram um estudo transversal com 100 crianças de seis a 12 anos e suas máes, assistidas pelo Programa de Saúde da Família (PSF) do município de Ribeirão Preto, e encontraram correlação entre instabilidade financeira e sintomas de hiperatividade e déficit de atenção nas crianças.

Vitolo, Fleitlich-Bilyk e Bordin (2005), em estudo realizado em Taubaté (SP), obtiveram resultados indicando que pertencer às classes econômicas $\mathrm{D} e$ E é um fator de risco para ocorrência de problemas de conduta, ansiedade/depressão e problemas de saúde mental geral na infância.

Assim, a literatura evidencia que as dificuldades socioeconômicas e a instabilidade financeira podem vir a influenciar no comportamento e desenvolvimento da criança, visto que essas dificuldades são acompanhadas de vários eventos estressores, afetando-as e aumentando a probabilidade da ocorrência de problema de saúde mental. Não obstante, torna-se fundamental destacar que a condiçáo socioeconômica não pode ser considerada variável única em um processo de desenvolvimento que é multideterminado (HALPERN; FIGUEIRAS, 2004; BANDIN, 2008).

Atuando conjuntamente com os fatores de risco, têm-se os fatores de proteção, que podem ser definidos como recursos pessoais ou sociais que amenizam ou inibem o impacto do risco (GREENBERG; DOMITROVICH; BUNBARGER, 2001). Os fatores de proteção podem atuar favorecendo o desenvolvimento humano, quando esse está sendo ameaçado pela exposiçáo ao risco e, portanto, podem ser identificados e ativados em situaçóes de risco (COIE et al., 1993; DEKOVIC, 1999; GREENBERG; DOMITROVICH; BUNBARGER, 2001).

Segundo Marturano (1999) e Schimitz (2003), as características do ambiente, quando positivas, podem contribuir como fatores protetivos ou estimuladores do desenvolvimento.

Mondin (2005) aponta alguns fatores de proteção existentes na família, como, por exemplo, redes de apoio social e relaçóes mais participantes e democráticas na família.

No Brasil existem alguns estudos que buscaram investigar aspectos relacionados à saúde mental 
infantil não só no que se refere aos fatores que podem ou náo estar associados com a presença e/ou ausência de problemas emocionais em crianças, como também às questôes epidemiológicas (FERRIOLI; MARTURANO; PUNTEL, 2007; PUNTEL, 2005; FLEITLICH; GOODMAN, 2000; CID, 2008).

Dentre as pesquisas que buscaram investigar a prevalência de transtornos emocionais na infância no Brasil, encontra-se o estudo desenvolvido por Fleitlich e Goodman (2004), os quais identificaram a prevalência de desordens emocionais em crianças de sete a 14 anos residentes em Taubaté, SP, no qual 1.251 crianças foram investigadas. Os autores encontraram a prevalência de $13 \%$ de transtornos psiquiátricos nessa população.

Paula, Duarte e Bordin (2007) investigaram a prevalência de problemas de saúde mental em crianças e adolescentes de seis a 17 anos em comunidade urbana de baixa renda, residentes em Embu, SP: 479 crianças/adolescentes participaram. Os resultados do estudo indicaram uma prevalência de $24,6 \%$ de problemas de saúde mental nas crianças e adolescentes.

Fleitlich (2005) aponta que o Brasil ainda não possui uma rede de suporte médico, psicológico e educacional adequada à demanda existente e que a divulgação de dados e informaçóes atuais e abrangentes sobre a saúde mental infantil pode facilitar o diálogo entre os educadores e profissionais da saúde assim como entre outros setores da sociedade.

Nessa direçáo, o presente estudo pretende dar continuidade ao estudo iniciado por Matsukura, Fernandes e Cid (2009), realizado junto a 39 responsáveis por crianças do primeiro ao quinto ano do ensino fundamental I de uma escola pública localizada na periferia de uma cidade de médio porte do interior do estado de Sáo Paulo. Os resultados desse estudo indicaram variáveis presentes no contexto familiar relacionadas com a presença de problemas de saúde mental na criança e outras foram relacionadas a melhores níveis como, por exemplo, o responsável brincar com a criança. Ao discutirem os resultados, as autoras apontam a complexidade dos processos de desenvolvimento e a necessidade de se aprofundar a compreensão sobre os diferentes contextos, práticas de cuidado e atores que se relacionam e que concretizam, no dia a dia, o que se denomina "de risco ou proteção" à saúde mental (MATSUKURA; FERNANDES; CID, 2009).

Assim, este estudo procurou ampliar essa avaliação, debruçando-se sobre as práticas e atividades do cotidiano dessas famílias que se encontram em situação de desvantagem socioeconômica.
Evidencia-se a necessidade do aprofundamento de investigaçóes dessa natureza a fim de compreender melhor os problemas de saúde mental infantil, bem como os fatores que a envolvem. Considera-se que esses estudos contribuem tanto com indicaçôes para continuidade de investigaçóes na área como também apresentando resultados para reflexóes que podem balizar possíveis açóes em relação à prevenção, implantação de serviços e intervençóes em saúde mental infantil.

\section{Objetivos}

Este estudo objetivou identificar e comparar situaçóes de risco ou proteçáo ao desenvolvimento socioemocional de crianças que vivem em contextos de desvantagem socioeconômica.

\section{Metodologia}

A partir do rastreamento da saúde mental das 39 crianças participantes do estudo de abordagem quantitativa, realizado anteriormente por Matsukura, Fernandes e Cid (2009), foram identificadas, conforme os escores do SDQ (GOODMAN, 1997), crianças com sintomas de saúde mental (de nível clínico), limítrofes e sem sintomas (normal)2 Nesse mesmo estudo, informaçôes acerca das famílias foram registradas, assim como investigadas dimensôes relativas às práticas parentais e atividades cotidianas da família, suporte social, dentre outras (MATSUKURA; FERNANDES; CID, 2009).

Nessa direção, o presente estudo, de caráter qualitativo, foi realizado buscando aprofundar a compreensão acerca da realidade vivenciada pelas crianças e famílias em evidenciada situação de risco, a partir de uma subamostra do estudo de Matsukura, Fernandes e Cid (2009). Assim, foram identificas famílias que possuem condiçôes socioeconômicas significativamente desfavoráveis, onde os responsáveis possuem baixa escolaridade, tendo cursado até o ensino fundamental II incompleto e, para a composiçáo dos grupos, consideraram-se as crianças, vinculadas a famílias assim caracterizadas, que apresentaram níveis clínicos de problemas de saúde mental (GSC) e que não apresentaram dificuldades dessa natureza na avaliação (GDT).

\subsection{Participantes}

Participaram deste estudo sete crianças, estudantes do ensino fundamental, com idade variando entre oito e 10 anos e seus responsáveis. Apresentam-se na 
Tabela 1 os dados gerais das crianças e responsáveis participantes do GSC e do GDT.

Conforme apontado, as crianças do presente estudo foram avaliadas através do Questionário de Capacidades e Dificuldade (SDQ) $)^{3}$ em estudo anterior realizado por Matsukura, Fernandes e Cid (2009) e por isso compóem os dois grupos, GSC e GDT.

Observa-se, a partir das informaçôes apresentadas na Tabela 1, que quatro crianças são do sexo feminino e três do sexo masculino e que as idades variam entre oito e 10 anos. Quanto à escolaridade, as crianças estáo distribuídas entre o segundo e o quinto ano do ensino fundamental. Apresentam-se na Tabela 2 os dados gerais dos responsáveis pelas crianças nos grupos com GSC e GDT.

Observa-se na Tabela 2 que a idade dos responsáveis participantes varia de 24 a 59 anos, sendo que dois deles não são as mães da criança. Quanto à escolaridade, a par com os critérios de composição dos grupos, essa variou do ensino fundamental I ao ensino fundamental II incompleto e a renda familiar não ultrapassou dois salários-mínimos.

\subsection{Local}

O estudo foi realizado em uma cidade de médio porte do estado de SP, em uma área periférica, designada como "zona crítica", a qual possui os maiores índices de vulnerabilidade social, destacando-se a extrema pobreza, altos índices de violência, desemprego, tráfico e consumo de drogas, prostituição infantil e baixa escolaridade da população (CAMPOS et al., 2003).

\subsection{Instrumentos de medida}

- 1) Roteiro de entrevista realizada junto ao responsável pela criança: Aborda 39 questôes relativas às características do responsável, composição e estrutura familiar, dificuldades enfrentadas no cotidiano, suporte social, relacionamento com filhos e familiares, cuidados parentais, características e informaçôes sobre as crianças.

- 2) Roteiro de entrevista realizado junto à criança: Aborda 35 questóes relativas às características das crianças, cotidiano, rotina, escola, relacionamento com membros da família e amigos, lazer, perspectivas para o futuro, dentre outras.

\subsection{Procedimentos}

O estudo foi aprovado pelo Comitê de Ética em Pesquisa em Seres Humanos da UFSCar sob protocolo n. 096/2011.

Tabela 1. Dados gerais das crianças participantes: GSC e GDT.

\begin{tabular}{ccccc}
\hline & \multicolumn{3}{c}{ GSC } & Número de irmãos \\
\hline Criança & Idade & Escolaridade & 7 \\
A - Menina & 10 & Quarto ano & 2 \\
B - Menina & 8 & Segundo ano & 4 \\
C - Menino & 9 & & Terceiro ano & 3 \\
D - Menino & 8 & & Terceiro ano & 3 \\
\hline & 9 & GDT & & 0 \\
\hline A - Menino & 10 & & Terceiro ano & 1 \\
B - Menina & 9 & Quinto ano & \\
C - Menina & & Quinto ano & \\
\hline
\end{tabular}

Tabela 2. Dados gerais dos responsáveis: GSC e GDT.

\begin{tabular}{cclcl}
\hline \multicolumn{5}{c}{ GSC } \\
\hline Responsável & Idade & \multicolumn{1}{c}{ Escolaridade } & Grau de parentesco & \multicolumn{1}{c}{ Renda familiar } \\
\hline A & 35 & Ensino fundamental II incompleto & Mãe & 2 salários-mínimos \\
B & 24 & Ensino fundamental II incompleto & Mãe & 1 salário-mínimo \\
C & 38 & Ensino fundamental II incompleto & Madrasta & 1 salário-mínimo \\
D & 39 & Ensino fundamental I & Mãe & 1 salário-mínimo \\
\hline \multicolumn{5}{c}{ GDT } \\
A & 59 & Ensino fundamental II incompleto & Avó & 1 salário-mínimo \\
B & 37 & Ensino fundamental II incompleto & Mãe & 2 salários-mínimos \\
C & 37 & Ensino fundamental II incompleto & Mãe & 2 salários-mínimos \\
\hline
\end{tabular}




\subsubsection{Construção e validação do roteiro da entrevista}

A partir de instrumento utilizado em estudo anterior (CID, 2008) e da revisão de literatura, elaboraram-se os dois roteiros de entrevista.

Para validação do instrumento, os roteiros foram submetidos à apreciação por um juiz especialista da área para verificar a adequação referente à linguagem, forma de realizaçáo das perguntas, sequência das questóes e adequação de abordagem sobre o fenômeno estudado, conforme recomenda a literatura (MANZINI, 2003).

Posteriormente foram realizadas aplicaçôes teste com duas crianças de oito e 10 anos e seus responsáveis. Através das aplicações teste observaram-se dificuldades dos entrevistados na compreensão de algumas questôes presentes na entrevista, dessa forma as questôes foram reformuladas.

Os temas abordados nas entrevistas foram: rotina, escola, descrição da criança, família, atividades familiares, facilidades e dificuldades da criança e do responsável, práticas de cuidado, brigas, discussóes e suporte social.

\subsubsection{Identificação e localização dos participantes}

Este estudo envolveu uma subamostra de participantes do estudo de Matsukura, Fernandes e Cid (2009). Os critérios para seleção dos atuais participantes foram: crianças avaliadas no estudo de Matsukura, Fernandes e Cid (2009) com sintomas clínicos de saúde mental (Grupo com Sintomas Clínicos de Saúde Mental - GSC) através do questionário de Capacidades e Dificuldades, e crianças avaliadas com desenvolvimento típico (Grupo com Desenvolvimento Típico - GDT), segundo o mesmo instrumento. Além disso, os participantes deveriam representar famílias classificadas como de baixa renda e baixa escolaridade (renda familiar de até três salários-mínimos e escolaridade do responsável até o ensino fundamental II incompleto) e residir na mesma periferia do município.

\subsubsection{Coleta de dados}

$\mathrm{Na}$ residência dos participantes foi explicado ao responsável os objetivos da presente pesquisa. Todos os envolvidos aceitaram participar, assim solicitou-se a assinatura do Termo de Consentimento Livre e Esclarecido. Os participantes responderam às entrevistas, que foram realizadas em mais de um encontro (média de dois). Primeiramente, as entrevistas foram realizadas com os responsáveis e, depois de um contato anterior para reconhecimento e apresentaçáo do pesquisador à criança, foi agendado o encontro para a realização da entrevista com ela.

\subsubsection{Análise de dados}

Quanto à análise de dados optou-se pela técnica do Discurso do Sujeito Coletivo (DSC), que é uma proposta de organização e tabulação de dados qualitativos de natureza verbal. Essa estratégia metodológica permite, através de uma estratégia discursiva, tornar clara uma dada representação social, na medida em que ela aparece sob a forma de um discurso que é a forma como os indivíduos expressam sua maneira de pensar (LEFÈVRE; LEFÈVRE, 2000).

As entrevistas foram transcritas na integra pela pesquisadora. Em seguida todas as questóes foram analisadas separadamente. Posteriormente, as expressóes chave, assim como as ideias centrais presentes na fala de cada um e também as comuns a todos relatos reunidos foram identificas. A partir daí elaborou-se o Discurso do Sujeito Coletivo, que visa construir, através das falas colhidas nas entrevistas, um discurso que represente determinado grupo social (LEFÈVRE; LEFÈVRE, 2000).

No presente estudo foram construídos, a saber:

1. DSC das crianças participantes;

2. DSC dos responsáveis pelas crianças.

\section{Resultados e discussão}

A seguir são apresentados alguns dos resultados obtidos por meio das análises dos DSCs dos dois grupos participantes.

Ressalta-se que os resultados e a discussão serão apresentados em duas partes, primeiramente os resultados relativos aos aspectos comuns presentes em ambos os grupos e, posteriormente, as divergências observadas entre os resultados do GSC e do GDT.

\subsection{Aspectos comuns identificados entre o GSC e o GDT}

Observa-se que os participantes, de ambos os grupos, relatam sobre a rotina, escola, família e apoio advindos de equipamentos de saúde, sociais, religiosos e educacionais de forma semelhante.

Em relação à rotina, identificou-se tanto no GDT como no GSC que as crianças possuem 
responsabilidades e regras definidas, determinadas pelos familiares, sendo que as regras envolvem responsabilidades domésticas, cumprimento de horários e obediência; como é possível observar a seguir, através do discurso das crianças de ambos os grupos referentes ao tema Rotina:

\section{Aham, quando sujo tenho que limpar, minha mãe manda eu arrumar a cama, que é a minha só (GDT - Crianças). Sim, eu tenho que cumprir horários, como não chegar tarde em casa, também tenho que fazer serviço de casa, não ir na rua, fazer o que meus familiares me pedem e não falar palavrôes (GSC - Crianças).}

A literatura indica que o estabelecimento de regras e responsabilidades é prática importante para um bom desenvolvimento emocional das crianças, por exemplo, o estudo de Ferriolli, Marturano e Puntel (2007) revelou que as crianças cujas famílias possuíam tal rotina apresentavam menos problemas de conduta que as crianças que náo possuem uma rotina estabelecida pelos cuidadores.

Quanto à escola, as crianças foram questionadas sobre quem as ajuda quando têm dificuldades na escola e todas apontaram que a ajuda vem unicamente da professora. Assim verifica-se a fundamental importância do papel do professor na vida dessas crianças, o qual aparece como única fonte de suporte em relação às dificuldades escolares percebida pelas crianças, destacando-se que os pais/responsáveis não foram citados por elas em ambos os grupos.

Pesquisas da área apontam para a importância do auxílio dos pais nas atividades escolares das crianças, uma vez que o suporte para a realizaçáo escolar se concretiza através do envolvimento direto dos pais com a vida acadêmica dos filhos. Sáo exemplos dessa modalidade de suporte a disposição de tempo e espaço adequado em casa para a realização dos deveres escolares, a exigência de cumprimento desses deveres, o intercâmbio regular com o professor e uma rotina de horários para as atividades diárias básicas (D'AVILA BACARJI; MARTURANO; ELIAS, 2005).

Não obstante, os resultados do presente estudo revelam que essas famílias não têm conseguido, no cotidiano, concretizar tais auxílios. Bastante compreensível, na medida em que muitos aspectos que envolvem tais práticas podem estar prejudicados, como, por exemplo, a falta de tempo, a impossibilidade de outra opçáo de prioridade entre lutar pela sobrevivência e organizar rotina e atenção, além da possibilidade da própria percepção do responsável acerca de sua capacidade de ajudar o filho. Em nossa amostra, por exemplo, o nível de escolaridade dos responsáveis era ter cursado o ensino fundamental II de forma incompleta. Nessa direção, a literatura aponta para a importância do auxílio dos pais nas atividades escolares das crianças, uma vez que o suporte para a realizaçáo escolar se concretiza por meio do envolvimento direto dos pais com a vida acadêmica dos filhos (D'AVILA BACARJI; MARTURANO; ELIAS, 2005).

Nessa medida, sugere-se a implementação e efetivação de canais de diálogo e intercâmbio com a escola e os professores, no sentido de esclarecer e debater sobre o papel deles como fatores protetivos no desenvolvimento da saúde mental dessas crianças.

Ainda que pese a grande demanda depositada sobre os professores, ressalta-se e reforça-se aqui a importância de instrumentalizá-los e apoiá-los para que esse profissional, de fato, possa oferecer às crianças oportunidades positivas de relacionamento e aprendizado, fundamentais para a oportunidade de desenvolvimento em pleno processo, como ocorre com crianças nessa faixa etária. Além disso, instrumentalizar professores e escola pode ser importante ferramenta no encaminhamento e estimulação junto a essa população. Aponta-se que a intersetorialidade deve ser prática exercida em todas as esferas em que criança e família estão inseridas.

Em relação ao tema Apoio, advindo de equipamentos sociais para o cuidado dos filhos e da família, observa-se nos discursos abaixo a presença de equipamentos de saúde, sociais, educacionais e religiosos:

\section{A capoeira (GDT - Responsável). Acho que a religião ajuda bastante (GDT - Responsável). $A h$, não posso dizer que o posto é um grande posto mais dá uma ajudada...tem o projeto das crianças, eles não ficam na rua, e tem o CRAS onde eu to aprendendo um monte de coisa" (GSC - Responsável).}

Em relação ao tema Atividades sociais de que a criança participa, destaca-se que, nos dois grupos, as crianças participam de programas e projetos existentes na comunidade, conforme mostram os discursos dos responsáveis apresentados a seguir:

Balé... capoeira... ela vai para a igreja, ajuda na sala das crianças (GDT - Responsável). Projeto no bairro que as crianças participam (GSC - Responsável).

Ressalta-se que nas famílias do GSC assim como nas famílias do GDT há preocupação, interesse e investimento da família na busca por recursos que possam auxiliar no cuidado da criança e no seu desenvolvimento. Leventhal e Brooks-Gunn 
(2000) relatam que famílias que procuram recursos na comunidade para promover o desenvolvimento de seus filhos podem contribuir efetivamente para melhores resultados, até mesmo em condiçóes desfavoráveis.

Assim, compreende-se que os resultados semelhantes encontrados no GSC e no GDT, como presença de responsabilidades na família, inserção em projetos e equipamentos sociais, podem ser considerados fatores protetivos da saúde mental das crianças, uma vez que estiveram concomitantemente atuando em ambiente de desvantagem socioeconômica, amenizando ou inibindo o impacto dos riscos existentes nesse contexto.

\subsection{Diferenças identificadas entre os grupos GSC e o GDT}

Em relação às diferenças encontradas entre o GSC e o GDT, observa-se que os grupos diferiram nos temas sobre escola, família, práticas de cuidado, brigas e discussóes, facilidades e dificuldades da criança, suporte advindo de equipamentos de saúde, sociais, religiosos e educacionais, dentre outros.

Em relação ao tema Escola, os discursos das crianças do GDT e do GSC apontam:

É boa... é muito legal estudar lá, cada professora tem um jeito de falar (GDT - Crianças). Chata (GSC - Crianças).

Além disso, quando foi indagado à criança como ela estava na escola, os discursos também diferiram:

Ah, eu vou bem (GDT - Crianças). Eu vou assim tudo bem, às vezes eu me sinto triste, algumas vezes sim (GSC - Crianças).

Observam-se diferenças nos discursos das crianças: no GSC as crianças apontam a escola de maneira negativa, sendo que nos momentos ruins elas se sentem tristes, diferentemente do GDT, no qual apenas aspectos positivos foram colocados.

A literatura aponta que as crianças com dificuldades socioemocionais apresentam maiores riscos para desenvolver dificuldades acadêmicas seguido de abandono escolar (FLEITLICH, 2005). Além disso, o fracasso escolar está intimamente ligado à situação emocional das crianças (BASTOS; ALMEIDA-FILHO, 1988).

No entanto, importa destacar que, devido ao processo de desenvolvimento ser dinâmico e multifatorial, é necessário refletir sobre o que é potencializado e de que forma um ciclo perverso, altamente prejudicial à criança, deve ser interrompido. Ou seja, as dificuldades socioemocionais implicam na defasagem escolar, na dificuldade de aprendizado e no desempenho escolar insatisfatório ou, seria o inverso; as dificuldades escolares da criança implicam nas dificuldades socioemocionais.

Nessa direção é necessário enfatizar a importância de intervir precocemente, visto que a prevenção e tratamento de transtornos mentais na infância e adolescência têm impacto no futuro, diminuindo o fracasso, abandono escolar e o desenvolvimento de transtornos na vida adulta (FLEITLICH; GOODMAN, 2002).

Referente ao tema Como eu sou, quando as crianças foram solicitadas a se descreverem, no GDT elas se descrevem relatando as atividades que realizam e citam aspectos positivos. Já no GSC, além de se descreverem positivamente, elas fazem referência sobre alguns aspectos envolvendo violência, conforme mostram os discursos:

Eu gosto de brincar... jogar bola, soltar pipa... Dançar (GDT - Criança). Eu sou legal, sou muito estimada, converso com outras pessoas... não gosto de ver as pessoas maltratando as outras, náo gosto de briga, quando vejo saio de perto (GSC - Criança).

Aponta-se que a convivência com a violência, no cotidiano familiar e/ou social, parece ser uma variável que quando presente em um contexto de risco focalizado torna o desenvolvimento socioemocional das crianças ainda mais vulnerável. Importa destacar que as crianças, ao falarem sobre seus limites e emoçóes, se apresentam bastante saudáveis e competentes no sentido de indicarem valores e/ ou limites importantes na maneira de se relacionar com as pessoas.

Pesquisas vêm indicando a associação entre vivenciar violência e sofrer problemas de saúde mental ao longo do ciclo de crescimento e desenvolvimento humano (ASSIS; AVANCI; OLIVEIRA, 2009; MARTINEZ; RICHTERS, 1993).

Em relação ao tema Sobre o que gosta e acha bom em sua vida, o discurso do GDT aponta que as crianças se referem aos relacionamentos com a família, amigos, além de brincar. Quanto às crianças do GSC, não relatam as relaçóes familiares, nem as amizades, apenas se referem ao brincar, inserção nos projetos e passeios, sem se referirem a relações sociais e/ou influências do núcleo familiar como referencial.

Minha mãe, conviver com os amigos, parentes, familia... brincar (GDT - Criança). Eu gosto mais de brincar... ir no projeto... andar de carro (GSC - Criança). 
Alguns autores distinguem a amizade como um dos aspectos que contribuem para a competência social da criança. Além disso, os processos de amizade promovem recursos emocionais e instrumentais que aumentam a capacidade de as crianças enfrentarem positivamente as demandas do ambiente, adaptando-se a ele (LADD; KOCHENDERFER; COLEMAN, 1996).

No entanto, ao não apontarem como positivo os relacionamentos (GSC) - seja de familiares ou de amigos - revela-se também a ausência do suporte social. O suporte social tem sido considerado uma medida de prevenção primária, necessária em todas as áreas da saúde (DALMASO; NEMES FILHO, 2001). Segundo Gutierres e Ribeiro (2002), o suporte social vem sendo indicado como um dos fatores que contribuem para o bem-estar, saúde física e mental dos indivíduos.

Não obstante, novamente questiona-se se a ausência do suporte social, assim como observado neste estudo em relação às dificuldades escolares, se a ausência antecede ou é resultado das dificuldades socioemocionais. Independentemente da resposta, há evidências suficientes para que ações na direção de efetivar e ampliar redes de suporte social das famílias e das próprias crianças sejam consideradas fundamentais para os processos de proteção frente à situação de risco aqui já estabelecida (COSTA; LUDEMIR, 2005; GUTIERRES; RIBEIRO, 2002).

Em relação ao tema Práticas de cuidado do responsável com as crianças, essas estiveram associadas à escola, às broncas e a brigas e discussóes.

Assim, quando questionado o responsável sobre o que ele faz quando a criança desobedece ou faz algo errado, os discursos apontaram:

$$
\begin{aligned}
& \text { Eu converso (GDT - Responsável). } \\
& \text { Eu sento o chinelo na bunda dele... } \\
& \text { eu castigo (GDT - Responsável). } \\
& \text { Nós brigamos, eu ponho de castigo, dou umas } \\
& \text { palmadas na bunda dele (GSC - Responsável). }
\end{aligned}
$$

Além disso, em relação às brigas e discussões familiares, no GDT os discursos apontam que nâo há brigas e discussôes, sendo que os conflitos envolvem somente diálogo. Diferentemente do GDT, no GSC os discursos mostraram que as brigas envolvem somente agressóes físicas, discussóes e que não há diálogo.

Assim, em ambos os grupos é possível observar a presença de castigos físicos, utilizando objetos como o chinelo ou a própria mão, e de brigas familiares envolvendo agressốes físicas e discussões.

Porém há uma diferença entre os grupos, pois no GDT os discursos apontaram a presença do diálogo, não sendo necessário o castigo físico, ou partir para a agressão física.

Esses resultados reforçam o que foi verificado tanto no estudo de Wille, Bettge e Sieberer (2008), realizado na Alemanha, como no estudo de Fleitlich e Goodman (2001), realizado no Brasil, que apontaram correlaçóes entre os altos índices de problemas de saúde mental infantil e violência e conflitos familiares.

Estudos apontam graves consequências relacionadas aos maus tratos na infância e os possíveis danos provocados por eles. Algumas das consequências apontadas são: baixa autoestima, agressividade, perda da confiança, dificuldade de aprendizagem, retraimento, falta de habilidades sociais (STERNBERG et al., 2006; SCHUMACHER; SLEP; HEYMAN, 2001).

Kohn et al. (2001) apontam que vários fatores advindos do ambiente familiar podem aumentar o risco de uma criança apresentar problemas emocionais, tais como a presença de conflitos entre os pais e a falta de interação entre eles.

Dentre os motivos pelos quais as brigas e discussões ocorrem, no GDT os motivos estão relacionados ao cotidiano familiar, como a desorganização da casa, contas a pagar, ou seja, questóes do dia a dia. Porém, no GSC, os discursos apontam que elas ocorrem devido ao relacionamento familiar e também devido ao uso de bebida alcoólica e falta de alimentação. Assim, observa-se que a dimensão das brigas e discussóes é diferente nos dois grupos, além de também diferir o que as causa.

Uma outra diferença observada entre os grupos foi verificada na temática Atividades da família, quanto às atividades que a criança realiza com o responsável durante a semana: observa-se que há uma diferença entre os dois grupos quanto à qualidade do compartilhamento das atividades, assim como com referência aos tipos de atividade que realizam. Nessa direção, os discursos referentes ao tema atividades familiares revelam:

\section{A gente bate um papo, fica conversando durante a semana (GDT - Criança). Eu ajudo a fazer o serviço, ajudo ela a lavar a louça, assisto televisão durante a semana (GSC - Criança).}

Observa-se que no GDT o discurso mostra que as crianças conversam com os responsáveis, já no GSC as crianças apontam que realizam os serviços domésticos e atividades como assistir TV, onde não há, necessariamente, uma interação entre o responsável e a criança durante a atividade. As crianças não apontaram, por exemplo, atividades onde diálogos ou interaçôes mais próximas, como passear ou brincar, ocorressem. 
Silva (2000) afirma que o diálogo com os filhos é fundamental no contexto educativo, pois está relacionado ao repertório inicial para o desenvolvimento de todas as habilidades sociais educativas. Nessa direção, algumas atividades, como, por exemplo, assistir televisão ou fazer as refeições conjuntamente, podem não implicar, necessariamente, em diálogo entre pais e filhos. Obviamente, não se trata aqui da ingenuidade de se considerar qualquer diálogo favorecedor de habilidades pura e simplesmente, porém o fato de a "conversa" existir e ser apontada pela criança parece indicar boas perspectivas nessa direção.

A literatura tem frequentemente apontado que aqueles pais que compartilham com a criança parte do seu tempo livre, proporcionando-lhe um elenco de atividades culturais e educacionais enriquecedoras, seja no lar seja na comunidade, favorecem o desenvolvimento cognitivo, o desempenho escolar e o ajustamento interpessoal (BRADLEY; CORWYN, 2002). Não obstante, ainda que o senso comum possa reproduzir a importância de interaçôes desse tipo, o cotidiano relatado por essas crianças não se caracteriza por essa realidade. Aponta-se que práticas educativas e oportunidades diversificadas a essa população poderiam favorecer e/ou estimular tais oportunidades de convívio.

Nessa direção, destaca-se que políticas públicas que direcionem oportunidades de lazer e integração entre membros de famílias inseridos na própria comunidade poderiam ser importantes oportunidades de desenvolvimento e manutenção da saúde mental de adultos e crianças.

O desafio colocado parece estar em como, enquanto profissionais compromissados com o desenvolvimento e a qualidade de vida dos envolvidos, realizar açóes e proposiçôes que, de fato, viabilizem tais práticas de lazer, que envolvam não só o fazer conjuntamente entre adultos e crianças ou entre crianças e seus pares, mas a troca de afeto, respeito, solidariedade e reconhecimento de individualidades tâo pouco consideradas no contexto onde estáo inseridas essas famílias.

A Terapia Ocupacional possui importantes ferramentas e recursos para intervir nessa direção. Nesse sentido, açóes junto a escolas, centros comunitários, nos projetos sociais, nos equipamentos de atenção básica ou mesmo junto aos responsáveis, em situaçóes diversas, parecem ser alternativas viáveis para intervençóes claramente voltadas para a promoção e prevenção da saúde mental dessa população.

\section{Considerações finais}

As divergências e semelhanças observadas entre os dois grupos focalizados no presente estudo permitem observar que alguns aspectos, embora apontados pela literatura como fatores de risco à saúde mental infantil, não foram aqui identificados, como por exemplo, a condiçáo socioeconômica desfavorável, a baixa escolaridade dos responsáveis, a presença de discussões na família e a prática de bater e castigar os filhos. Não obstante, quando diferenças foram identificadas, elas encontravam-se mais na esfera de ausência de possibilidades advindas do cuidador ou, em outras palavras, de sua incapacidade de fornecer à criança formas mais afetivas e positivas de interação em um cotidiano já marcadamente difícil. Assim é que atividades de lazer muito limitadas, compartilhamento de atividades envolvendo pouca interação afetiva, presença de violência doméstica, dentre outras, parecem fazer parte do cotidiano das crianças que se tornaram vulneráveis ${ }^{4}$ ao risco vivenciado no cotidiano.

Neste estudo, a partir das consideraçóes do referencial teórico existentes sobre risco e proteção e saúde mental infantil, podemos compreender que as crianças do grupo GDT são resilientes ${ }^{5}$ e as crianças do grupo GSC, vulneráveis (RUTTER, 1987, 1999; COIE et al., 1993). Não obstante considera-se que este estudo contribui para a identificação e compreensão das variáveis envolvidas no processo de desenvolvimento socioemocional de crianças avaliadas com problemas de saúde mental e de crianças que não possuem tais dificuldades e que vivenciam situações semelhantes.

Além disso, o estudo pode contribuir para o debate acerca das políticas públicas e programas efetivos para atender a todas as crianças e adolescentes que necessitem de atençáo a saúde mental e aqui aponta-se como fundamental a implementação de políticas, serviços de saúde mental e práticas preventivas nessa área voltados para a população de crianças e jovens.

É fundamental destacar o limite do estudo em relação ao pequeno número de participantes; nesse sentido aponta-se que os resultados e discussão devem ser considerados a luz dessa condição.

\section{Referências}

ASSIS, S. G.; AVANCI, J. Q.; OLIVEIRA, R. V. C. de. Desigualdades socioeconômicas e saúde mental infantil. Revista de Saúde Pública, São Paulo, v. 43, p. 92-100, 2009. Suplemento 1.

BANDIN, J. A criança em situação de pobreza extrema. In: ASSUMPÇÃO JUNIOR, F.; KUCZYNSKI, E. Situaçôes psicossociais na infância e adolescência. São Paulo: Atheneu, 2008. p. 113-123. 
BASTOS, A. C.; ALMEIDA-FILHO, N. Determinação social da saúde mental infantil: revisão da literatura epidemiológica. Psicologia: Teoria e Pesquisa, Brasília, v. 4, n. 3, p. 268-282, 1988.

BRADLEY, R. H.; CORWYN, R. F. Socioeconomic status and child development. Annual Review of Psychology, Palo Alto, v. 53, n. 1, p. 371-399, 2002. PMid:11752490. http://dx.doi.org/10.1146/annurev.psych.53.100901.135233 CAMPOS, S. E. A. et al. O lazer cotidiano do Jardim Gonzaga - São Carlos. In: ENCONTRO NACIONAL DE RECREAÇĀO E LAZER, 15, 2003, Santo André. Anais... Santo André: SESC, 2003. CD-ROM.

CID, M. F. B. O impacto da saúde mental materna: estilo parental, suporte social e desenvolvimento sócio-emocional dos filhos. 2008. Dissertação (Mestrado em Educação Especial)-Universidade Federal de São Carlos, São Carlos, 2008.

COIE, J. D. et al. The science of prevention: a conceptual framework and some directions for a National Research Program. American Psychologist, Durham, v. 48, n. 10, p. 1013-1022, 1993. http://dx.doi. org/10.1037/0003-066X.48.10.1013

COSTA, A. G.; LUDEMIR, A. B. Transtornos mentais comuns e apoio social: estudo em comunidade rural da zona da mata de Pernambuco, Brasil. Cadernos de Saúde Pública, Rio de Janeiro, v. 21, n. 1, p. 73-79, 2005.

CURY, C. R.; GOLFETO, J. H. Strengths and difficulties questionnaire (SDQ): a study of school in Ribeirão Preto. Revista Brasileira de Psiquiatria, São Paulo, v. 25, n. 3, p. 139-145, 2003.

DALMASO, A. S. W.; NEMES FILHO, A. A. Promoção à saúde. Brasília: IDS, 2001.

D'AVILA-BACARJI, K. M. G.; MARTURANO, E. M.; ELIAS, L. C. S. Suporte parental: um estudo sobre crianças com queixas escolares. Psicologia em Estudo, Maringá, v. 10, n. 1, p. 107-115, 2005.

DEKOVIC, M. Risk and protective factors in the development of problem behavior during adolescence. Journal of Youth and Adolescence, New York, v. 28, n. 6, p. 667-685, 1999. http://dx.doi. org/10.1023/A:1021635516758

FERRIOLI, S. H. T.; MARTURANO, E. M.; PUNTEL, L. P. Contexto familiar e problemas de saúde mental infantil no Programa Saúde da Família. Revista de Saúde Pública, São Paulo, v. 41, n. 2, p. 251-259, 2007. http:// dx.doi.org/10.1590/S0034-89102006005000017

FLEITLICH, B. A saúde nos jovens brasileiros: quais são os principais problemas e como podemos ajudar? Jornal Brasileiro de Psiquiatria, Rio de Janeiro, v. 54, n. 3, p. 168-169, 2005.

FLEITLICH, B.; GOODMAN, R. Epidemiologia. Revista Brasileira de Psiquiatria, São Paulo, v. 22, n. 2, p. 2-6, 2000.

FLEITLICH, B. W.; GOODMAN, R. Social factors associated with child mental health problems in Brazil: cross sectional survey. British Medical Journal, London, v. 323, n. 7313, p. 599-600, 2001. http://dx.doi. org/10.1136/bmj.323.7313.599
FLEITLICH, B. W.; GOODMAN, R. Implantação e implementação de serviços de saúde mental comunitários para crianças e adolescentes. Revista Brasileira de Psiquiatria, São Paulo, v. 24, n. 1, p. 2, 2002.

FLEITLICH, B.; GOODMAN, R. Prevalence of child and adolescent psychiatric disorders in southeast Brasil. Journal of the American Academy of Child and Adolescent Psychiatry, Baltimore, v. 42, n. 6, p. 728-734, 2004.

GOODMAN, R. The strengths and difficulties questionnaire: a research note. Journal of Child Psychology and Psychiatry, Malden, v. 38, n. 5, p. 581-586, 1997. http://dx.doi.org/10.1111/j.1469-7610.1997.tb01545.x

GREENBERG, M. T.; DOMITROVICH, C.; BUMBARGER, B. The prevention of mental disorders in school-aged children: current state of the field. Prevention \& Treatment, Washington, v. 4, n. 1, 2001. http://dx.doi. org/10.1037/1522-3736.4.1.41a

GROTBERG, E. H. Introdução: novas tendências em resiliência. In: MELILLO, A.; OJEDA, E. N. S. Resiliência: descobrindo as próprias fortalezas. Porto Alegre: Artmed, 2005. p. 15-22.

GUTIERRES, M. C.; RIBEIRO, J. L. Suporte social e qualidade de vida em pessoas com perturbaçōes mentais crônicas apoiadas por serviços comunitários. Lisboa: INR, 2002.

HALPERN, R.; FIGUEIRAS, A. C. M. Influências ambientais na saúde mental da criança. Jornal de Pediatria, Porto Alegre, v. 80, n. 2, p. 104-110, 2004. http://dx.doi. org/10.1590/S0021-75572004000300013

HUTZ, C. S. Violência e risco na infância e adolescência: pesquisa e intervenção. 1. ed. São Paulo: Casa do Psicólogo, 2005.

KOHN, R. et al. Factores de riesgo de trastornos conductuales y emocionales en la nińez: estudio comunitario en el Uruguay. Revista Panamericana de Salud Publica, Washington, v. 9, n. 4, p. 211-218, 2001. PMid:11418967. http://dx.doi.org/10.1590/S1020-49892001000400002

KOLLER, S. H. Ecologia do desenvolvimento humano. São Paulo: Casa do Psicólogo, 2004.

LEFÈVRE, F.; LEFÈVRE, A. M. C. Os novos instrumentos no contexto da pesquisa qualitativa. In: LEFÈVRE, F.; LEFÈVRE, A. M. C.; TEIXEIRA, J. J. V. (Orgs.). O discurso do sujeito coletivo: uma nova abordagem metodológica em pesquisa qualitativa. Caxias do Sul: EDUCS, 2000. p. 11-35.

LEVENTHAL, T.; BROOKS-GUNN, J. The neighborhoods they live in: the effects of neighborhood residence upon child and adolescent outcomes. Psychological Bulletin, Washington, v. 126, n. 2, p. 309-337, 2000. PMid:10748645. http://dx.doi. org/10.1037/0033-2909.126.2.309

LADD, G. W.; KOCHENDERFER, B. J.; COLEMAN, C. C. Friendship quality as a predictor of young children's early school adjustment. Child Development, Chicago, v. 67, n. 3, p. 1103-1118, 1996. PMid:8706512. http:// dx.doi.org/10.2307/1131882

LINHARES, M. B. M.; BORDIM, M. B. M.; CARVALHO, A. E. V. Aspectos do desenvolvimento psicológico da criança ex-prematura na fase escolar. 
In: MARTURANO, E. M.; LINHARES, M. B. M.; LOUREIRO, S. R. (Orgs.). Vulnerabilidade e proteção: indicadores na trajetória de desenvolvimento escolar. São Paulo: Casa do Psicólogo; FAPESP, 2004. p. 75-106. (Cap. 3).

MANZINI, E. J. Considerações sobre a elaboração de roteiro para entrevista semi-estruturada. In: MARQUEZINE, M. C.; ALMEIDA, M. A.; OMOTE, S. (Orgs.). Colóquios sobre pesquisa em educação especial. Londrina: EDUEL, 2003. p. 11-25. (v. 1).

MARTINEZ, P. E.; RICHTERS, J. E. Violent communities, family choices, and children's chances: an algorithm for improving the odds. Development and Psychopathology, New York, v. 5, n. 4, p. 609-627, 1993. http://dx.doi.org/10.1017/S0954579400006192

MARTURANO, E. M. Recursos no ambiente familiar e dificuldades de aprendizagem na escola. Psicologia: Teoria e Pesquisa, Brasília, v. 15, n. 2, p. 135-142, 1999. http://dx.doi.org/10.1590/S0102-37721999000200006

MATSUKURA, T. S.; FERNANDES, A. D. S. A.; CID, M. F. B. Fatores de risco e proteçâo à saúde mental infantil: atividades de lazer e práticas de cuidado dos pais com as crianças. São Carlos: UFSCar, 2009. (Relatório de pesquisa de Iniciação Científica PIBIC/CNPq).

MONDIN, E. M. C. Um olhar ecológico da família sobre o desenvolvimento humano. Psicologia Argumento, Curitiba, v. 23, n. 41, p. 25-35, 2005.

PAULA, C. S.; DUARTE, C. S.; BORDIN, I. S. Prevalence of mental health problems in children and adolescents from the outskirts of São Paulo city: treatment needs and service capacity evaluation. Revista Brasileira de Psiquiatria, São Paulo, v. 29, n. 1, p. 11-17, 2007.

POLETTO, M.; WAGNER, T. M. C.; KOLLER, S. H. Resiliência e desenvolvimento infantil e crianças que cuidam de crianças: uma visão em perspectiva. Psicologia: Teoria e Pesquisa, Brasília, v. 10, n. 3, p. 241-250, 2004.

PUNTEL, L. P. Problemas emocionais e de comportamento em crianças de 6 a 12 anos cadastradas em um Núcleo de Atenção Primária e Saúde da Família. 2005. 70 f. Dissertação (Mestrado)-Universidade de São Paulo, Ribeirão Preto, 2005.
RUTTER, M. Psychosocial resilience and protective mechanisms. American Orthopsychiatric Association, Greenville, v. 57, n. 3, p. 316-331, 1987.

RUTTER, M. Resilience concepts and findings: implications for family therapy. Journal of Family Therapy, Oxford, v. 21, n. 2, p. 119-144, 1999. http://dx.doi. org/10.1111/1467-6427.00108

SAPIENZA, G.; PEDRÔMONICO, M. R. M. Risco, proteção e resiliência no desenvolvimento da criança e do adolescente. Psicologia em Estudo, Maringá, v. 10, n. 2, p. 209-216, 2005.

SCHIMITZ, M. F. Influences of race and family environmental on child hyperactivity and antisocial behavior. Journal of Marriage \& the Family, Menasha, v. 65, n. 4, p. 835-849, 2003. http://dx.doi. org/10.1111/j.1741-3737.2003.00835.x

SCHUMACHER, J. A.; SLEP, A. M. S.; HEYMAN, R. E. Risk factors for child neglect. Aggression and Violent Behavior, New York, v. 6, p. 231- 254, 2001. http://dx.doi. org/10.1016/S1359-1789(00)00024-0

SILVA, A. T. B. Problemas de comportamento e comportamentos socialmente adequados: sua relação com as habilidades sociais educativas de pais. 2000. $145 \mathrm{f}$. Dissertação (Mestrado)-Universidade Federal de São Carlos, São Carlos, 2000.

STERNBERG, K. J. et al. Effects of early and later family violence on children's behavior problems and depression: a longitudinal, multi-informant perspective. Child Abuse \& Neglect, Oxford, v. 30, n. 3, p. 283-306, 2006. PMid:16524627. http://dx.doi.org/10.1016/j. chiabu.2005.10.008

VITOLO, Y. L. C.; FLEITLICH-BILYK, R. G.; BORDIN, I. A. S. Crenças e atitudes educativas dos pais e problemas de saúde mental em escolares. Revista de Saúde Pública, São Paulo, v. 39, n. 5, p. 716-724, 2005. WILLE, N.; BETTGE, S.; SIEBERER, U. R. Risk and protective factors for children's and adolescent's mental health: results of the BELLA study. European Child \& Adolescent Psychiatry, Toronto, v. 17, p. 133-147, 2008. Supplement 1. 


\section{Contribuição dos Autores}

Thelma Matsukura e Maria Fernanda Cid: redação do texto e revisão. Amanda Fernandes: elaboraçáo, redação e revisão do texto.

\section{Fonte de Financiamento}

Apoio de Bolsa de Iniciação Científica do Programa CNPq/PIBIC/UFSCar.

\section{Notas}

${ }^{1}$ A pesquisa foi submetida e aprovada pelo Comitê de Ética em Pesquisa em Seres Humanos da UFSCar, sob protocolo n. 096/2011.

${ }^{2}$ O SDQ avalia as cinco subescalas que avaliam hiperatividade, sintomas emocionais, problemas de conduta, relaçóes interpessoais e comportamento pró-social e o total das dificuldades através da somatória de pontos que resultaram nos escores clínico, limítrofe e normal.

${ }^{3}$ O SDQ é um questionário que rastreia problemas de saúde mental infantil, contendo um total de 25 itens divididos em cinco subescalas: problemas emocionais, hiperatividade, relacionamento, conduta e comportamento pró-social, com cinco itens em cada subescala (CURY; GOLFETO, 2003).

${ }^{4}$ Vulnerabilidade é a predisposição individual para o desenvolvimento de doenças ou de comportamentos “ineficazes" em situação de crise ou na presença de risco. Um indivíduo pode ser identificado como vulnerável quando diante de eventos de risco seu desenvolvimento é prejudicado. Sendo assim, alguns indivíduos - com diferenças fisiológicas, psicológicas, contextuais/ambientais - são mais suscetíveis ou vulneráveis a certos eventos negativos, quando comparados a outras pessoas na mesma situação de risco (POLETTO; WAGNER; KOLLER, 2004).

${ }^{5}$ Habilidade de superar adversidades, o que não significa que o indivíduo saia da crise ileso, como implica o termo invulnerabilidade (GROTBERG, 2005). 